\title{
Acid-Fastness of Histoplasma in Surgical Pathology Practice
}

\author{
Madhu Rajeshwari · Immaculata Xess ${ }^{1}$ \\ Mehar Chand Sharma D Deepali Jain
}

Departments of Pathology and ${ }^{1}$ Microbiology, All India Institute of Medical Sciences, New Delhi, India

Received: May 10, 2017

Revised: June 27, 2017

Accepted: July 11, 2017

Corresponding Author

Deepali Jain, MD, DNB, FIAC

Department of Pathology, All India Institute of

Medical Sciences, Ansari Nagar, New Delhi 110029,

India

Tel: +91-9868895112

Fax: $+91-11-26588663$

E-mail: deepalijain76@gmail.com
Background: Histoplasmosis (HP) is diagnosed by visualizing intracellular microorganisms in biopsy and/or culture. Periodic-acid Schiff (PAS) and Gomori methenamine silver (GMS) staining methods are routinely used for identification. The acid-fast property of Histoplasma was identified decades ago, but acid-fast staining has not been practiced in current surgical pathology. Awareness of the acid-fast property of Histoplasma, which is due to mycolic acid in the cell wall, is important in distinguishing Histoplasma from other infective microorganisms. Here, we examined acid-fastness in previously diagnosed cases of Histoplasma using the Ziehl-Neelsen (ZN) stain and correlated those findings with other known fungal stains. Methods: All cases diagnosed as HP were retrieved and reviewed along with $\mathrm{ZN}$ staining and other fungal stains. We also stained cases diagnosed with Cryptococcus and Leishmania as controls for comparison. Results: A total of 54 patients ranging in age from 11 to 69 years were examined. The most common sites of infection were the skin, adrenal tissue, and respiratory tract. Of the total 43 tissue samples, 20 (46.5\%) stained positive with the ZN stain. In viable cases, a significant proportion of microorganisms were positive while necrotic cases showed only rare ZN-positive yeasts. In comparison to PAS and GMS stains, there was a low burden of ZN-positive yeasts. Cryptococcus showed characteristic ZN staining and all cases of Leishmania were negative. Conclusions: Although the morphology of fungal organisms is the foundation of identification, surgical pathologists should be aware of the acid-fast property of fungi, particularly when there is the potential for confusion with other infective organisms.

Key Words: Histoplasma; Acid fast; Ziehl-Neelsen; Fungal organisms
Histoplasmosis (HP) is a major systemic fungal infection caused by two varieties of Histoplasma. H. capsulatum var. capsulatum, which causes the classic form of the disease, and var. duboisii, which causes African HP. ${ }^{1}$ Despite the availability of serological and molecular tests, the gold standard for the diagnosis of the majority of fungal infections is demonstration of the organisms, either in tissue sections/aspirate smears, or by culture. ${ }^{2}$ However, fungal culture is time consuming (a minimum of 15 days for Histoplasma) and the materials for culture may not be available in all cases. ${ }^{2}$ Hence, histochemical staining for fungi can play a pivotal role in the timely diagnosis of fungal infections.

Gomori methenamine silver (GMS) and periodic-acid Schiff (PAS) are the two most commonly used broad-spectrum fungal stains in routine histopathology practice. These stains help to distinguish fungi based on morphologic characteristics such as size, type of budding, presence of hyphae, and branching. Histoplasma are characterized as $2-4 \mu \mathrm{m}$ in size with round to oval uninucleate yeast cells that may show narrow-based budding. A clear space or artifactual halo may be apparent around the microorganisms due to retraction of the basophilic fungal cell cyto- plasm from the poorly stained cell wall. They are usually intracellular, clustered within the histiocytes. Nonetheless, other yeast forms of similar size may be difficult to discriminate from one another. Histoplasma may be confused with capsule deficient Cryptococcus neoformans, Candida glabrata, Leishmania donovani, Penicillium marneffei, Sporothrix schenckii, the small form of Blastomyces dermatitidis and endospores of Coccidioides spp. ${ }^{3}$

Narrow spectrum fungal stains can help to solve the differentiation and identification problem of yeasts, the most well known being the mucicarmine stain, Alcian Blue and Fontana-Masson stains for Cryptococcus, and Congo red for Blastomyces. ${ }^{4}$ The ZiehlNeelsen $(\mathrm{ZN})$ stain, commonly used to identify the mycobacteria, is a less known narrow spectrum fungal stain. Although the acid-fast property of Histoplasma was identified decades ago, it has never been used routinely for the diagnosis of HP. We propose that $\mathrm{ZN}$ staining can be used for the identification of fungal organisms, especially Histoplasma. Here, we aimed to examine acid-fastness in previously diagnosed cases of Histoplasma by ZN staining and the results were compared with PAS and GMS stains. We also stained 10 cases each of Leishmania and Cryptococcus infection, 
the two most common morphologic mimickers, as controls for the $\mathrm{ZN}$ stain.

\section{MATERIALS AND METHODS}

All cases of HP diagnosed between 2010 and 2016 were retrieved from the Department of Pathology archives. The study was approved by the Institutional Ethics Committee (No. IEC-564/ 2.12.2016) and performed in accordance with the principles of the Declaration of Helsinki. Written informed consents were obtained. Hematoxylin and eosin, PAS, and GMS stained slides were reviewed and diagnosis reconfirmed. Cases with adequate tissue in paraffin blocks were selected and a modified $\mathrm{ZN}$ staining procedure was performed by the Kinyoun method. The acid-fastness of fungi was characterized in the $\mathrm{ZN}$ stains in all the cases.

The modified ZN staining procedure (Kinyoun method) was performed as follows. Paraffin-embedded sections were deparaffinized with xylene, rehydrated with graded concentrations of alcohol, and brought to water. The slides were flooded with carbol fuchsin for 30 minutes, washed, and then decolorized with $1 \%$ acid alcohol. The slides were then counterstained with $1 \%$ methylene blue. The number of $\mathrm{ZN}$-positive yeasts was counted per 100 identified yeast cells. For comparison, ZN staining was performed in selected cases diagnosed as cryptococcosis $(n=10)$ and leishmaniasis $(n=10)$ and the results were compared with cases of HP. There were no cases of coccidioidomycosis or blastomycosis in our archive for comparison.

\section{RESULTS}

There were a total of 66 tissue samples from 54 patients diagnosed with HP during the study period. Adequate tissue for ZN staining was available only in 43 samples from 37 patients. Patients (33 men, four women) had an age range of 11 to 69 years. All of the specimens were from biopsies except for one case of intestinal resection. Culture/serology details were available in 18 cases, of which eight (44\%) were positive. For rest of the cases, morphology was taken as the gold standard method of diagnosis. Immune status was known in 31 cases, of which 11 (35.5\%) were immunocompromised with human immunodeficiency virus (HIV) infection and diabetes as the most common causes of immunosuppression. The most common sample site was the skin (37\%) followed by adrenal tissue (23\%) and the respiratory tract $(11.6 \%$, Table 1). Apart from the lungs, there was involvement of the nasopharynx, vocal cords, and trachea in one case each. Miscellaneous sites of involvement included bone marrow $(n=3)$, buc-
Table 1. Summary of clinical details of all patients

\begin{tabular}{lc}
\hline Parameter & No. \\
\hline Total No. of HP samples diagnosed during study period & 66 \\
ZN stain & 43 (37 patients) \\
Sex, $n$ (\%) & \\
$\quad$ Males & $33(89.2)$ \\
$\quad$ Females & $4(10.8)$ \\
Age (yr) & $11-69$ \\
Sites (\%) & \\
Skin & 37 \\
Adrenal & 23 \\
Lung and respiratory tract & 11.6 \\
Others & 28.4 \\
Disseminated cases (\%) & 32.4 \\
Immunocompromised state (\%) & 35.5 \\
Culture/Serology positivity (\%) & 44 \\
ZN positivity (\%) & 46.5 \\
Culture positive cases that showed ZN positivity, $\mathrm{n}(\%)$ & $4 / 8(50)$ \\
\hline
\end{tabular}

$\mathrm{HP}$, histoplasmosis; ZN, Ziehl-Neelsen.

cal mucosa $(n=1)$, tongue $(n=1)$, orbit $(n=1)$, lymph node $(n=1)$, ileum $(n=1)$, and retroperitoneum $(n=1)$. Fever, weight loss, pancytopenia and molluscum-like papules were common clinical presentations. Some of the rare presentations included subacute intestinal obstruction with ileal perforation, ulcerative lesion in the tongue mimicking a vesiculobullous lesion, tumor-like growth in the trachea and nasopharyngeal involvement in one case each. Twelve cases had disseminated disease (32.4\%), four of which had more than one tissue sample available. Adrenal, skin, and bone marrow involvement were common in these disseminated cases. Two of the patients with disseminated HP were immunosuppressed (HIV-positive). Both of these cases had a rapid clinical course, which was fatal in one case.

Of the 43 samples, 20 (46.5\%) stained positive with the ZN procedure. The number of yeasts that showed positivity varied from less than $1 \%$ to $20 \%$ (Fig. 1A-D). Among the eight culture and serology positive cases, four exhibited $\mathrm{ZN}$-positive staining. On morphology, four cases showed entirely necrotic tissue. In comparison to the entirely necrotic cases, viable cases showed more $\mathrm{ZN}$-positive microorganisms. Out of the four necrotic cases, only one case showed $\mathrm{ZN}$-positive yeasts. In comparison to PAS and GMS stains, ZN-positive yeasts were low in burden (Figs. 2A-C, 3A, B).

In contrast to Histoplasma, the cryptococcal yeasts displayed a peculiar staining pattern (Fig. 4A, B) where the capsule and cell wall stained a granular magenta to purple color. This pattern of staining could easily be differentiated from the crisp bright pink cytoplasmic staining of Histoplasma. Almost all of the yeasts in all of the cases stained uniformly, which made the cells easy to identify 

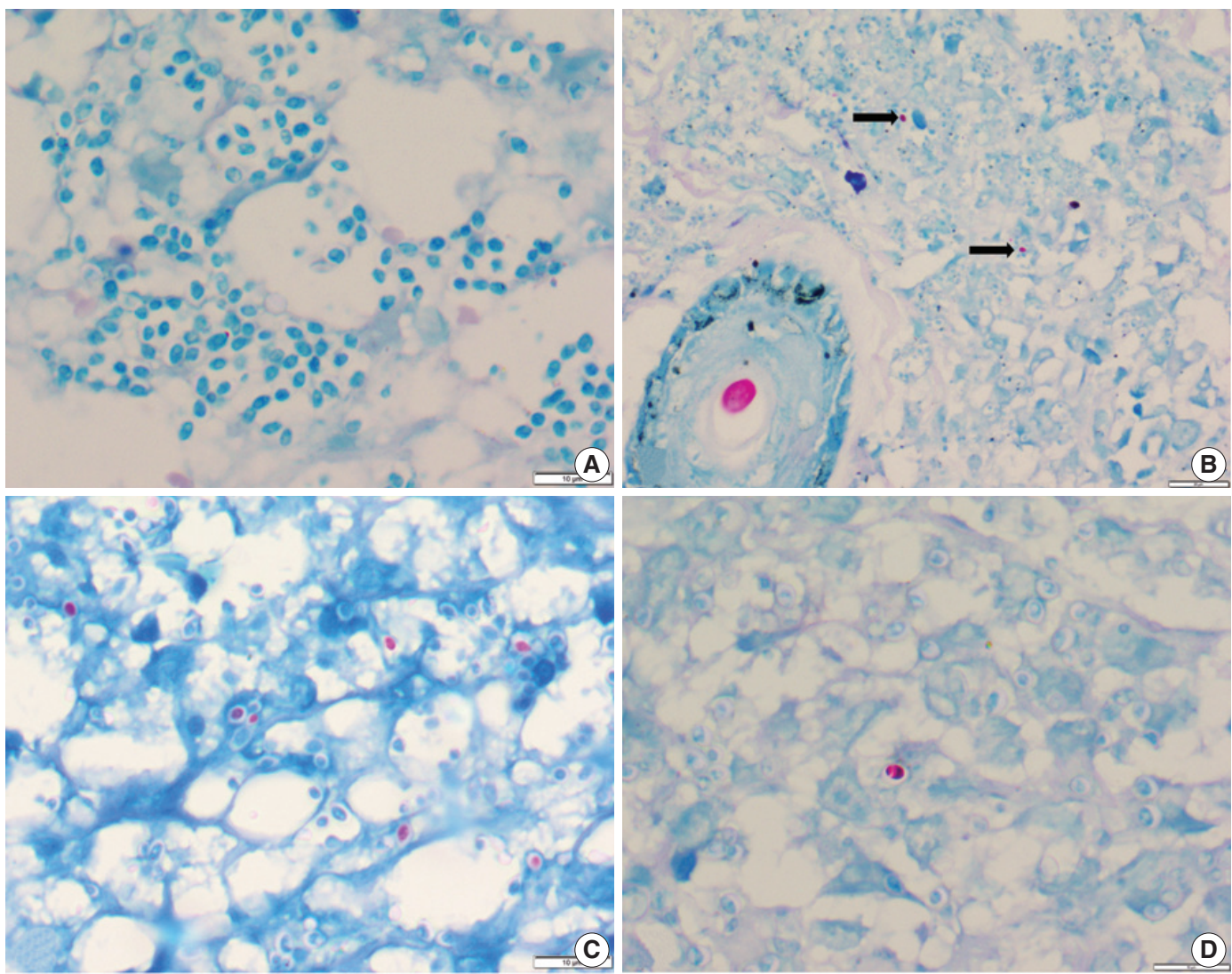

Fig. 1. (A) Photomicrograph of a histoplasmosis case showing Ziehl-Neelsen (ZN)-negative yeasts. (B) Skin biopsy showing ZN-positive Histoplasma yeast cells (arrows); ZN-positive hair shaft in a hair follicle is shown for color comparison. (C, D) Two different cases show numerous to few ZN-positive yeast cells.
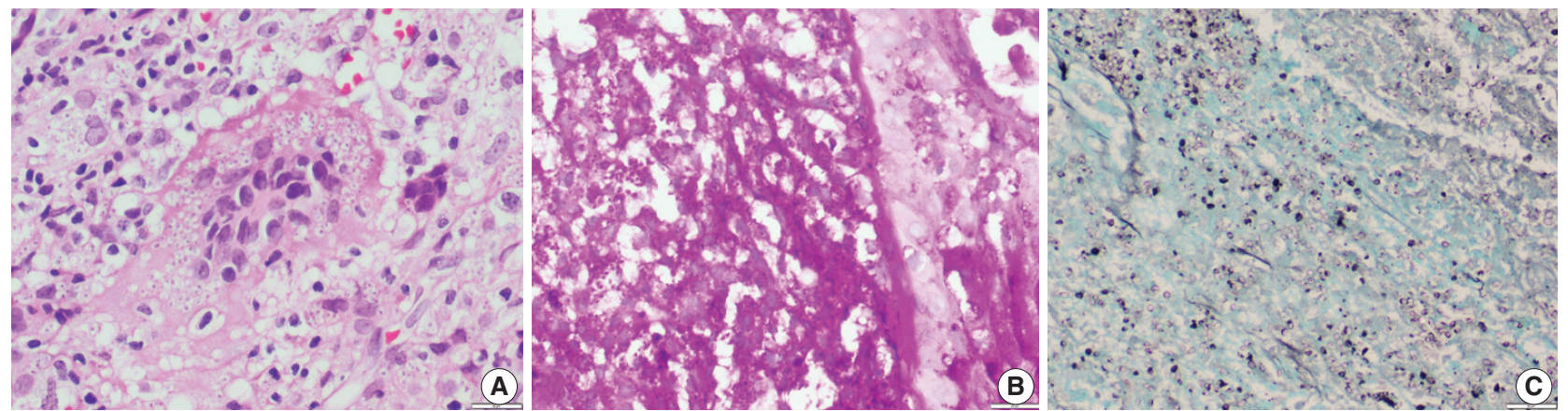

Fig. 2. (A) Photomicrograph shows numerous extracellular and intracellular Histoplasma yeast cells present within a giant cell. Histoplasma are round to oval in shape with an eccentric purple dot or crescent. (B, C) Periodic-acid Schiff and Gomori methenamine silver stains highlight fungal profiles.

even under low power objective microscopy. None of the cases of leishmaniasis showed positivity for $\mathrm{ZN}$ staining.

\section{DISCUSSION}

HP, initially thought to be endemic in the Eastern USA and
Latin America, is being increasingly recognized in Asian countries like India with the majority of cases being reported from Eastern and North-Eastern regions. ${ }^{1} \mathrm{~A}$ total of 38 cases were reported from India until 1996. ${ }^{5}$ Another series from India reported 24 cases during a span of 10 years. ${ }^{6}$ Because our hospital is a tertiary institute, we recorded 54 cases in six years, making it the largest 

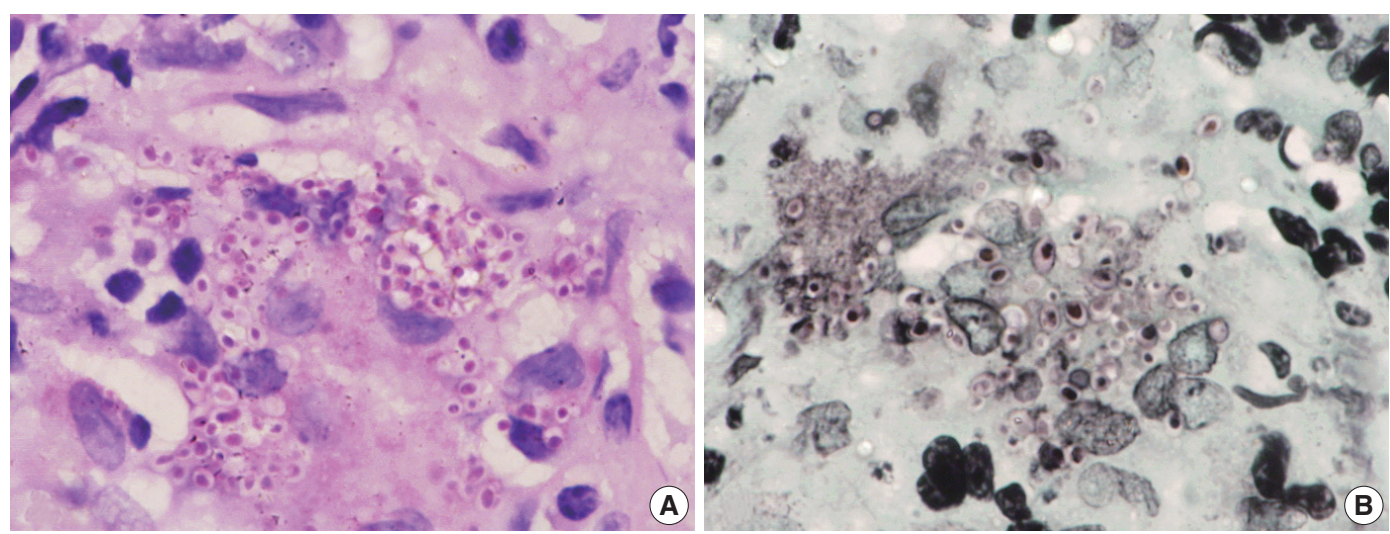

Fig. 3. Oil immersion magnification of periodic-acid Schiff (A) and Gomori methenamine silver (B) Histoplasma stains.

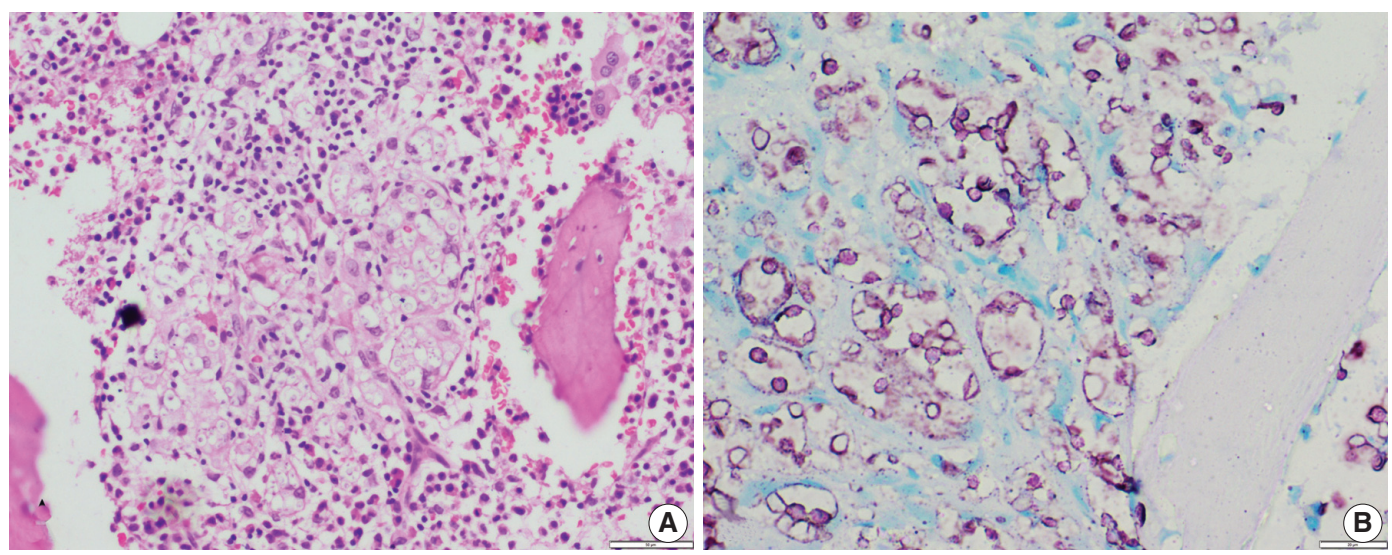

Fig. 4. (A) A case of cryptococcal infection involving bone marrow shows numerous fungal organisms that are round in shape and surrounded by a clear space. (B) Ziehl-Neelsen staining highlights all cryptococci and surrounding capsular halos with magenta color.

Table 2. Morphological mimics of Histoplasma

\begin{tabular}{ll}
\hline Morphological mimic & \\
\hline Capsule deficient Cryptococcus & Size variation, weak positivity for mucin stains and positivity for FM stain; acid fast (present study) \\
Leishmania & Presence of kinetoplast \\
Small variant of Blastomyces & Broad-based budding \\
Candida albicans & Presence of pseudohyphae \\
Candida glabrata & More size variability, neutrophilic reaction \\
Penicillium marneffei & Presence of transverse septum \\
Endospores of Coccidioides & Presence of intact/ruptured spherules \\
Histoplasma & Round to oval intracellular yeasts with narrow based budding and surrounded by a halo, acid-fast ZN positive \\
\hline
\end{tabular}

FM, Fontana Masson; ZN, Ziehl-Neelsen.

series of HP from the Indian subcontinent. This large increase in cases indicates that the incidence of HP is increasing in India in recent times. HP is no longer a disease of immunocompromised patients; ${ }^{7}$ in our series, only one-third of the patients had a known risk factor.

Classic diagnostic methods include microscopy, culture, antigen detection by enzyme immunoassay, antibody detection by complement fixation and immunodiffusion, and polymerase chain reaction assays. ${ }^{7}$ The gold standard methods for the diagnosis of HP includes the demonstration of yeasts on microscopy and isolation of the mold by culture. ${ }^{7,8}$ Although culture of the organism should always be sought and attempted, it is most effective only in cases of high fungal burden with chronic or disseminated forms of HP and culturing is often insensitive in sub-acute, acute, and mild forms of $\mathrm{HP}^{2}$ Time constraints for culture combined with a lack of specificity and sensitivity make histopatho- 
logical examination an easy, rapid, and reliable diagnostic method.? We have used morphology as the gold standard for the diagnosis of HP where cultures were negative or unavailable. Histoplasma are characterized as $2-4 \mu \mathrm{m}$ in size with round to oval uninucleate cells that are usually surrounded by a halo. However, many similar sized yeast varieties may be difficult to differentiate from Histoplasma (Table 2).

Parsons and Zarafonetis ${ }^{10}$ first observed acid-fastness in Histoplasma as early as 1945 , even before the discovery of GMS. Rawson $^{11}$ also found that the acid-fast property could be advantageously used to search for Histoplasma. Although acid-fastness of Histoplasma came to light decades ago, its utility in routine diagnostic practice has not been explored. In our study, nearly $46.5 \%$ of the samples were positive for $\mathrm{ZN}$ staining. The number of yeasts that were acid-fast varied from less than $1 \%$ to $20 \%$. This is consistent with the one previous study available, where the authors noted that $47 \%$ of Histoplasma cases were acid-fast. ${ }^{12}$ Similarly, only a few to one-third of yeast forms showed this property in their study as well as our previous reported case included in this series. ${ }^{12,13}$ Until now, none of the fungi other than Histoplasma and Blastomyces were known to be acid-fast, and this property can be used to advantage in severely necrotic cases where the yeast forms may be scant and may be erroneously diagnosed as other common diseases like tuberculosis (TB). This is especially important in TB endemic countries such as India where we receive most of the cases of $\mathrm{TB}$ in routine practice and $\mathrm{ZN}$ staining is commonly performed for the diagnosis of TB. Therefore, knowledge about the ZN positivity of Histoplasma may be beneficial in recognizing yeast forms in necrotic cases of HP.

Acid-fast microorganisms are characterized by wax-like, nearly impermeable cell walls that contain mycolic acid and large amounts of fatty acids and complex lipids. Treatment with hot hydrochloric acid abolishes the acid-fastness of Histoplasma, suggesting that this property of Histoplasma is likely due to the presence of mycolic acid in the cell wall. However, Blastomyces resist this decolorization, signifying that fatty acids other than mycolic acid are responsible for acid-fastness. ${ }^{12}$ We use a modified $\mathrm{ZN}$ stain in our laboratory for the diagnosis of TB and leprosy in routine services where the concentration of phenol (mordent) in carbol fuchsin was increased from $5 \%$ to $8 \%$, which contributes to better stain penetration.

The acid-fastness of Histoplasma could not be demonstrated in cytology smears in our previous study. ${ }^{14}$ Instead, Histoplasma yeasts were identified easily against the pale background of $\mathrm{ZN}$ stain in necrotic cases. However, the case numbers were too small to draw any conclusions and more studies are needed on aspirate smears. We have also observed the peculiar pattern of positivity of cryptococcal yeasts with the $\mathrm{ZN}$ stain. To the best of our knowledge, this finding has not been reported previously. In contrast to Histoplasma, almost all of the yeasts showed uniform positivity, making it one of the most sensitive and specific stains for the identification of cryptococci. Since the pattern of $\mathrm{ZN}$ positivity is different, this feature might also help to differentiate Cryptococcus and Histoplasma in combined infections, which is relatively common in immunosuppressed hosts. Endospores of Coccidioides resemble those of Histoplasma; however, we do not see Coccidioides in India so the comparison could not be made in this study.

In conclusion, this study is one of the largest series of HP where the acid-fastness of Histoplasma was evaluated. A comparative analysis of Cryptococcus and Leishmania revealed a different pattern of acid-fastness. Although morphology is the basis of fungal identification, a simple $\mathrm{ZN}$ stain may be included in the diagnostic armamentarium for fungal infections in surgical pathology. Although every organism is not acid-fast within a single case, approximately half of $\mathrm{HP}$ cases are positive on $\mathrm{ZN}$ staining. The difference in ZN positivity staining pattern between Histoplasma and Cryptococcus may serve as a useful distinguishing feature. Surgical pathologists should be aware of the acid-fast property of fungal organisms, which will be helpful in the correct identification of the microorganism and ultimately patient management in morphologically-challenging HP cases.

\section{Conflicts of Interest}

No potential conflict of interest relevant to this article was reported.

\section{Acknowledgments}

The abstract of the same work has been accepted for presentation in 2017 USCAP Annual Meeting; 4-10 Mar 2017; San Antonio, TX, USA.

\section{REFERENCES}

1. Antinori S. Histoplasma capsulatum: more widespread than previously thought. Am J Trop Med Hyg 2014; 90: 982-3.

2. Guimarães AJ, Nosanchuk JD, Zancopé-Oliveira RM. Diagnosis of histoplasmosis. Braz J Microbiol 2006; 37: 1-13.

3. Guarner J, Brandt ME. Histopathologic diagnosis of fungal infections in the 21st century. Clin Microbiol Rev 2011; 24: 247-80.

4. Youngberg GA, Wallen ED, Giorgadze TA. Narrow-spectrum histo- 
chemical staining of fungi. Arch Pathol Lab Med 2003; 127: 1529-30.

5. Goswami RP, Pramanik N, Banerjee D, Raza MM, Guha SK, Maiti PK. Histoplasmosis in eastern India: the tip of the iceberg? Trans R Soc Trop Med Hyg 1999; 93: 540-2.

6. Gopalakrishnan R, Nambi PS, Ramasubramanian V, Abdul Ghafur $\mathrm{K}$, Parameswaran A. Histoplasmosis in India: truly uncommon or uncommonly recognised? J Assoc Physicians India 2012; 60: 25-8.

7. Kauffman CA. Histoplasmosis: a clinical and laboratory update. Clin Microbiol Rev 2007; 20: 115-32.

8. Azar MM, Hage CA. Laboratory diagnostics for histoplasmosis. J Clin Microbiol 2017; 55: 1612-20.

9. Joseph Wheat L. Current diagnosis of histoplasmosis. Trends Microbiol 2003; 11: 488-94.
10. Parsons RJ, Zarafonetis CJ. Histoplasmosis in man: report of seven cases and review of 71 cases. Arch Intern Med 1945; 75: 1-23.

11. Rawson AJ. Acid-fast property of Histoplasma capsulatum. Am J Clin Pathol 1948; 18: 97.

12. Wages DS, Wear DJ. Acid-fastness of fungi in blastomycosis and histoplasmosis. Arch Pathol Lab Med 1982; 106: 440-1.

13. Jain D. Acid fast property of histoplasma: a concept revitalized. Int J Surg Pathol 2016; 24: 724-5.

14. Ranjan R, Jain D, Singh L, Iyer VK, Sharma MC, Mathur SR. Differentiation of histoplasma and cryptococcus in cytology smears: a diagnostic dilemma in severely necrotic cases. Cytopathology 2015; 26: $244-9$. 